OnLine Journal of Biological Sciences 12 (2): 62-71, 2012

ISSN 1608-4217

(C) 2012 S. RayChaudhuri et al., This open access article is distributed under a Creative Commons Attribution

(CC-BY) 3.0 license

\title{
Isolation of Nitrate and Phosphate Removing Bacteria from Various Environmental Sites
}

\author{
${ }^{1}$ DebRoy, S., ${ }^{1}$ S. Das, ${ }^{1}$ S. Ghosh, ${ }^{1}$ S. Banerjee, \\ ${ }^{1}$ D. Chatterjee, ${ }^{1}$ A. Bhattacharjee, ${ }^{2}$ I. Mukherjee and ${ }^{1}$ Shaon RayChaudhuri \\ ${ }^{1}$ Department of Biotechnology, \\ School of Biotechnology and Biological Sciences, \\ ${ }^{2}$ Department of Natural Sciences, School of Mangement and Sciences; \\ West Bengal University of Technology, BF-142, \\ Sector-1, Salt Lake, Kolkata-700064, West Bengal, India
}

\begin{abstract}
Problem statement: Nitrate and phosphate are two major pollutants due to anthropogenic activity like excessive use of fertilizers in agriculture. Their contamination has emerged as a global problem and its potential threat is marked on the environmental sustainance as well as on the public health. Approach: The objective of the current study is to isolate efficient nitrate and phosphate removing microbes from various environmental sites that have been selected on the basis of the nature of polutants received by them and their water quality assessment. These well characterized isolates could in future be used for the remediation of waste water. 30 different sites were screened using culture based method. The nitrate and phosphate removing abilities of the microbes were checked in enriched medium (Himedia M439) after $16 \mathrm{~h}$ of incubation at $37^{\circ} \mathrm{C}$. Results: 7 efficient isolates were obtained from rhizosphere of Water lily, Marine beaches, Paddy field and Raw sewage canal. The highest nitrate removal (88.3\%) was shown by isolate (WBUNB009) from raw sewage canal and the highest phosphate removal (82.9\%) was shown by isolate (WBUNB004) from rhizosphere of Water lily. Morphologically all the isolates were gram positive bacilli as reconfirmed by environmental scanning electron microscopy. Biochemically as well as physiologically they differ from each other. Conclusion/Recommendation: This study leads to the isolation of efficient nitrate and phosphate removers from environmental origin. The phosphate removing efficiency is much higher than the type strain under identical condition. These native microbes might be responsible for maintaining the phosphate and nitrate levels at the 30 sites investigated inspite of the received pollution load. These isolates could be the potential bioremedial agents for other sites with high nitrate and phosphate contamination level.
\end{abstract}

Key words: Nitrate, phosphate, agricultural runoff, bacteria, Eutrophication, bioremediation, efficient isolates, phosphate contamination, European Community (EC)

\section{INTRODUCTION}

Nitrate and Phosphate are recognized as the major nutrients which are required by living organisms for their physiological processes. They are most commonly added as fertilizer to enhance the quality of soil. However they have emerged as most abundant pollutants in the world due to their excess usage. The traditional agricultural practices like dry farming with marginal irrigation, flood plain farming and random application of fertilizers are considered as diffused sources of nitrate and phosphate in soil and aquifers. Besides this, the irregular rainfall during different seasons and the stream flow pattern causes seepage of these contaminants from soil to surface and ground water (Whitmore et al., 1992; Jorgensen, 1999; Giupponi et al., 1999; Agrawal, 1999; Krishnaswamy et al., 2009).

The cultivation patterns like terrace farming results in nitrate leaching into aquifers (Nakasone and Yamamoto, 2004; Kinoshita et al., 2003).

Increased levels of nitrate up to $400 \mathrm{ppm}$ have been detected in groundwater (Filintas et al., 2008). Possible sources of nitrate pollution include manure, agricultural fertilizer, industrial effluent, domestic wastewater, septic systems, human waste lagoons, animal feedlots and native soil organic matter, as well as geologic Corresponding Author: Shaon RayChaudhuri, Department of Biotechnology, School of Biotechnology and Biological Sciences, West Bengal University of Technology, BF-142, Sector-I, Saltlake. Kolkata-700064, India 
sources (Jin et al., 2004) Other point sources of nitrate are municipal sewage canals, septic tanks, sewage dumping grounds (Wakida and Lerner, 2005; 2006). The mining tailings, industrial effluent from nuclear reactors, radioactive waste processing units mainly those dealing with compounds like plutonium or thorium nitrate (Singleton et al., 2005). Nitrate contamination is a global problem and stands as second most dangerous pollutant after the pesticides. High concentration of nitrate in drinking water is a threat especially to infants, causing methemoglobinemia, also called "blue baby syndrome. The carcinogenic effect of nitrate is also reported. Concentration higher than $10 \mathrm{ppm}$ in drinking water may also cause stomach cancer in infants (Jin et al., 2004). EPA has demarcated the maximum contaminant level to be $10 \mathrm{ppm}$ for $\mathrm{NO}_{3}$ $\mathrm{N}$ and $45 \mathrm{ppm}$ for $\mathrm{NO}_{3}$ concentration. A similar guideline of $50 \mathrm{ppm}$ as $\mathrm{NO}_{3}$ has been set by the WHO and the European Community (EC). Several conventional technologies adopted for nitrate removal are ion exchange resins, electro dialysis, reverse osmosis and distillation which substantially increase the cost of operation. Therefore the cost-effective alternative lies in the biological denitrification process (Pinar et al., 1997; Eckford and Fedorak, 2002).

The addition of phosphorus as phosphate fertilizers in soil in excessive amount causes serious environmental problems in the form of eutrophication which uses up large amounts of oxygen. The main sources of phosphate in aquatic environment is through household sewage water containing detergents and cleaning preparations, agricultural effluents containing fertilizers as well as industrial effluent from fertilizer, detergent and soap industries (Pradyot, 1997). Phosphate is generally present as polyphosphate and orthophosphate. The concentration of phosphate in water bodies vary from $0.005-10 \mathrm{ppm}$ depending on the source of phosphate near the water body. On one hand digestive problems occur from extremely high levels of phosphate, on the other, phosphate levels greater than 1.0 may interfere with coagulation in water treatment plants. The EPA has fixed standard phosphate levels as $0.015 \mathrm{ppm}$ for water supply, $0.025 \mathrm{ppm}$ for aquatic life, $0.05 \mathrm{ppm}$ for lakes and $0.02 \mathrm{ppm}$ for mountain lakes (Kotoski, 1997). Microbial strategies are currently being used for the removal of excess phosphate load in waste water since it is an attractive alternative to chemical processing (Krishnaswamy et al., 2009).

The objective of the present study is to isolate efficient nitrate and phosphate reducing microbes from different environmental water bodies. Further characterization of these isolates would lead to the development of an array of novel organisms which can reduce nitrate and phosphate load in water bodies of various environmental sites leading to bioremediation.

\section{MATERIALS AND METHODS}

Sampling: Water samples were collected from different environmental sites to screen for nitrate and phosphate removing organisms. The sites were selected on the basis of the pollutants received by them. Main focus was on sites, which according to pollutants received were expected to have high load of nitrate and phosphate but showed low concentrations of these. Presumably these are the sites which host the nitrate and phosphate removers. The list of sites has been given below Table 1 .

Environmental parameters: Different physical, chemical and biological parameters were assessed for the water samples from each site to understand the different concentration of pollutants in them. The physical and chemical parameters were assessed as per the protocol laid down by Central Pollution Control board of India. The biological parameter of the sites were assessed by studying the normal population of the sites. 10 microbes were selected which are commonly present in waste water (viz Escherichia coli, Enterobacter aerogenes, Shigella flexneri, Klebsiella pneumonia, Pseudomonas aeruginosa, Proteus mirabilis, Enterococcus faecalis, Salmonella sp. and Staphylococcus aureus.) and the water from the sites were serially diluted and spread on media specific for the microbes. The different media used were HiChrome E.coli coliform Selective agar base (HiMedia M-1294 for Escherichia coli, Enterobacter aerogenes, Shigella flexneri, Klebsiella pneumonia); HiChrome UTI agar base (HiMedia M-1353 for Pseudomonas aeruginosa, Proteus mirabilis, Enterococcus faecalis); Salmonella differential agar base (HiMedia M-1078 for Salmonella sp) and HiChrome Aureus agar base (HiMedia M-1468 for Staphylococcus epidermidis and Listeria monocytogenes).

Cultivation medium and growth conditions: Since one of the main objectives of our study is to isolate nitrate reducing microbes therefore the screening for microbes were done in high nitrate containing medium. The water samples of all the above mentioned sampling sites were serially diluted and plated on media containing 2000ppm of nitrate followed by overnight incubation at $37^{\circ} \mathrm{C}$ to isolate microbes which can 
OnLine J. Biol. Sci., 12 (2): 62-71, 2012

Table 1:Water samples were collected from the following sites. The sampling sites were selected on the basis of the nature of the pollutatnts received by them. The co-ordinates of the sampling sites have been mentioned below

\begin{tabular}{|c|c|c|c|}
\hline Types & Locations & CO-Ords & Source of waste \\
\hline \multirow[t]{6}{*}{ Marine coast } & Udaypur Beach & $21^{\circ} 36^{\prime} 31^{\prime \prime} \mathrm{N}$ & Recreational activity, animal waste \\
\hline & (West Bengal) & $87^{\circ} 28^{\prime} 52^{\prime \prime} \mathrm{E}$ & in low volume, natural waste. \\
\hline & Digha Beach & $21^{\circ} 36^{\prime} 59^{\prime \prime} \mathrm{N}$ & Recreational activity, animal \\
\hline & (West Bengal) & $87^{\circ} 30^{\prime} 08^{\prime \prime} \mathrm{E}$ & waste, natural waste. \\
\hline & Mandarmani Beach & $21^{\circ} 41^{\prime} \mathrm{N}$ & Recreational activity, \\
\hline & (West Bengal) & $87^{\circ} 33^{\prime} \mathrm{E}$ & animal waste, natural waste. \\
\hline \multirow{4}{*}{$\begin{array}{l}\text { Artificial water } \\
\text { Bodies }\end{array}$} & Haldiram fountain & $22^{\circ} 37^{\prime} 40^{\prime \prime} \mathrm{N}$ & Bathing, natural waste, \\
\hline & & $88^{\circ} 26^{\prime} 01^{\prime \prime} \mathrm{E}$ & washing activity. \\
\hline & Baguiati fountain & $22^{\circ} 36^{\prime} 36^{\prime \prime} \mathrm{N}$ & Natural waste \\
\hline & & $88^{\circ} 25^{\prime} 41^{\prime \prime} \mathrm{E}$ & \\
\hline \multirow[t]{8}{*}{ River } & Ganga (Naihati) & $\begin{array}{l}22^{\circ} 53 ’ 23 ’ \mathrm{~N} \\
88^{\circ} 24^{\prime} 40^{\prime \prime} \mathrm{E}\end{array}$ & $\begin{array}{l}\text { Industrial waste, idol immersion, domestic waste, recreational waste, } \\
\text { oil spill from transportation, }\end{array}$ \\
\hline & Ichamati (Taki) & $\begin{array}{l}22^{\circ} 36^{\prime} 01^{\prime \prime} \mathrm{N} \\
88^{\circ} 56^{\prime} 46^{\prime \prime} \mathrm{E}\end{array}$ & $\begin{array}{l}\text { piggery waste, sewage waste from surrounding area. } \\
\text { Industrial waste, idol immersion, domestic waste, } \\
\text { recreational waste, agricultural waste, erosion of banks }\end{array}$ \\
\hline & Churni (Ranaghat) & $23^{\circ} 14^{\prime} 35^{\prime \prime} \mathrm{N}$ & Human bathing, domestic and sewage waste, \\
\hline & & $88^{\circ} 36^{\prime} 23^{\prime \prime} \mathrm{E}$ & transportation by boat, agricultural waste, industrial waste. \\
\hline & Damodar (Bardhaman) & $23^{\circ} 12^{\prime} 41^{\prime \prime} \mathrm{N}$ & Bathing, recreational waste, domestic waste, \\
\hline & & $87^{\circ} 50^{\prime} 48^{\prime \prime} \mathrm{E}$ & agricultural waste, fish aquaculture, oil spillage from launch \\
\hline & Mayurakshi (Tilpara) & $23^{\circ} 57^{\prime} 04$ ”N & Fishing, washing, small \\
\hline & & $87^{\circ} 31^{\prime} 06^{\prime} \mathrm{E}$ & scale agricultural run off \\
\hline \multirow[t]{9}{*}{ Hotspring: } & Agnikundu, Bakreswar & $23^{\circ} 52,51$ ”, & Rice and coins thrown by \\
\hline & & $\begin{array}{l}87^{\circ} 22^{\prime} 32^{\prime \prime} \mathrm{E} \\
23^{\circ} 52,51, \mathrm{~N}\end{array}$ & devotees, plastic packets, \\
\hline & Dudhkundu, Bakreswar & $\begin{array}{l}23^{\circ} 52,51^{\prime \prime N} \\
87^{\circ} 22^{\prime} 34^{\prime \prime} \mathrm{E}\end{array}$ & Natural waste, rice and coins. \\
\hline & Jibatsakundu, Bakreswar & $23^{\circ} 52^{\prime} 52^{\prime \prime} \mathrm{N}$ & Algal growth seen, natural waste, \\
\hline & & $87^{\circ} 22^{\prime} 34^{\prime \prime} \mathrm{E}$ & rice and coins by devotees. \\
\hline & Swet ganga (Birbhum) & $23^{\circ} 52^{\prime} 52^{\prime \prime} \mathrm{N}$ & Washing of hands and feet, \\
\hline & & $87^{\circ} 22^{\prime} 34^{\prime \prime} \mathrm{E}$ & waste from rituals e.g., flowers, utensil washing. \\
\hline & Taptapani (Orissa) & $19^{\circ} 29^{\prime} 04^{\prime \prime} \mathrm{N}$ & Human bathing, natural \\
\hline & & $84^{\circ} 23^{\prime} 37^{\prime \prime} \mathrm{E}$ & $\begin{array}{l}\text { wastes, rice and coins } \\
\text { thrown by devotees. }\end{array}$ \\
\hline \multirow[t]{2}{*}{ Paddy field } & Berunanpukuria (Barasat) & $22^{\circ} 44^{\prime} 34^{\prime \prime} \mathrm{N}$ & Fertilizer waste, \\
\hline & & $88^{\circ} 26^{\prime} 20^{\prime \prime} \mathrm{E}$ & agricultural run off. \\
\hline Rhizosphere & Typha sp & $22^{\circ} 35^{\prime} 14^{\prime \prime} \mathrm{N}$ & Dumping of wastes from the nearby region. \\
\hline of Swampy & Aquatic grass & $88^{\circ} 28^{\prime} 31^{\prime \prime} \mathrm{E}$ & $\begin{array}{l}\text { (beside the ONGC green building), } \\
\text { animal grazing therefore }\end{array}$ \\
\hline \multirow[t]{3}{*}{ Plants } & $\begin{array}{l}\text { Nymphaeae nouchali } \\
\text { (violet flower, water lily) }\end{array}$ & & animal waste \\
\hline & Water Hyacinth & $22^{\circ} 35^{\prime} 40^{\prime \prime} \mathrm{N}$ & Bathing, dumping of waste \\
\hline & & $88^{\circ} 23^{\prime} 51^{\prime \prime} \mathrm{E}$ & $\begin{array}{l}\text { from local shops and residence, washing of utensils, } \\
\text { cleaning of buses and trucks, }\end{array}$ \\
\hline Natural water & Under Ultadanga over & $22^{\circ} 41^{\prime} 19^{\prime \prime} \mathrm{N}$ & Domestic waste dumping from \\
\hline \multirow[t]{13}{*}{ Bodies } & bridge (Kolkata) Kalyani & $88^{\circ} 25^{\prime} 05^{\prime \prime} \mathrm{E}$ & local residence, erosion of banks. \\
\hline & $\begin{array}{l}\text { Byepass } \\
\text { Expressway (Fordillapur) }\end{array}$ & & \\
\hline & Site reserved for Himangini & $22^{\circ} 43^{\prime} 34^{\prime \prime} \mathrm{N}$ & Aquaculture, dumping of waste from \\
\hline & & $88^{\circ} 24^{\prime} 12^{\prime \prime} \mathrm{E}$ & local residence, erosion of banks. \\
\hline & Jute Retting Site & $22^{\circ} 47^{\prime} 12^{\prime \prime N}$ & Used for jute retting, \\
\hline & Thankurbari & $88^{\circ} 30^{\prime} 12^{\prime \prime} \mathrm{E}$ & dumping of waste from local shops and residence \\
\hline & Kaikhali pond & $22^{\circ} 38,03 ” \mathrm{~N}$ & Bathing, washing clothes, \\
\hline & & $88^{\circ} 26^{\prime}, 16^{\prime \prime E}$ & cleaning utensils, idol immersion. \\
\hline & Rajarhat pond & $\begin{array}{l}22^{\circ} 35^{\prime} 52^{\prime \prime} \mathrm{N} \\
88^{\circ} 28^{\prime} 09^{\prime \prime} \mathrm{E}\end{array}$ & $\begin{array}{l}\text { Animal waste, Pisciculture, } \\
\text { recreational activity, erosion of the banks. }\end{array}$ \\
\hline & Captain Bheri & $22^{\circ} 33^{\prime} 10^{\prime \prime} \mathrm{N}$ & Sewage, domestic and garage waste \\
\hline & & $88^{\circ} 24^{\prime} 43^{\prime \prime} \mathrm{E}$ & $\begin{array}{l}\text { passed through a crude filtration for removal of coarsensuspended } \\
\text { solids. Primarily used for pisciculture. Bathing and cleaning } \\
\text { also found, agricultural run-off from surrounding region. }\end{array}$ \\
\hline & Private Bheri & $22^{\circ} 33^{\prime} 19^{\prime \prime} \mathrm{N}$ & \\
\hline & & $88^{\circ} 24^{\prime} 41^{\prime \prime} \mathrm{E}$ & \\
\hline \multirow{6}{*}{$\begin{array}{l}\text { Raw sewage } \\
\text { Canal }\end{array}$} & Kestopur khal & $22^{\circ} 35^{\prime} 50^{\prime \prime} \mathrm{N}$ & Domestic and sewage waste, \\
\hline & & $88^{\circ} 25^{\prime} 37^{\prime \prime} \mathrm{E}$ & $\begin{array}{l}\text { waste from agricultural } \\
\text { land, oil from garage }\end{array}$ \\
\hline & Bagjola khal & $22^{\circ} 37^{\prime} 16^{\prime \prime} \mathrm{N}$ & Domestic and sewage waste, \\
\hline & & $88^{\circ} 24^{\prime} 10^{\prime \prime} \mathrm{E}$ & $\begin{array}{l}\text { waste from agricultural land, } \\
\text { oil from garage, effluent } \\
\text { from rubber industry. }\end{array}$ \\
\hline & Khal Next to & $22^{\circ} 33^{\prime} 17^{\prime \prime} \mathrm{N}$ & Sewage, domestic and garage waste. \\
\hline & Captain Bheri & $88^{\circ} 24^{\prime} 47^{\prime \prime} \mathrm{E}$ & Dumping from local area. \\
\hline
\end{tabular}


Further selection was made on the basis of morphology. The cultures were re-streaked three or more times to obtain pure colonies. The isolates were also grown in Nitrate Broth (Himedia M439-500G) and maintained at $37^{\circ} \mathrm{C}$ in $150 \mathrm{rpm}$ shaking condition. The final selection of the pure isolates was on the basis of their nitrate removing efficiency from the liquid culture.

Morphological characterization: The initial morphology of the isolates were determined by using light microscope (1000X magnification on a Zeiss Axiostar Plus microscope) following simple staining using 5\% Crystal Violet. The Gram nature of the isolate was determined by differential staining as per standard procedure.

Dimensions of the isolates were determined using Environmental scanning electron microscopy (FEI QUANTA 200 MARK 2 at $15 \mathrm{kV}$ ) as per the protocol.

Biochemical characterization: The ability of the isolates to produce enzymes like DNase, oxidase, lipase, catalase and amylase was determined. The tests for the first five enzymes were done according to the protocol of Nandy et al. (2007) the amylase test was done on $1 \%$ starch agar plate and incubated at $37^{\circ} \mathrm{C}$ for overnight followed by flooding of the plate with iodine solution. The substrate utilization profile of the isolates were checked as per manufacturer's protocol using substrate utilization kits (HiMedia KB009).

Antibiotic assay: The response of the isolates towards 18 different antibiotics (HiMedia) were checked according to the procedure reported by Nandy et al. (2007)

Nitrate removal: The isolates were inoculated $(2 \%$ inoculum) in Nitrate broth and incubated for $16 \mathrm{~h}$ at $37^{\circ} \mathrm{C}$ in a shaking incubator $150 \mathrm{rpm}$. The cell free supernatant was taken for estimation of nitrate removal after harvesting the culture at $8609 \times \mathrm{g}$ for $10 \mathrm{~min} .200 \mu \mathrm{L}$ of Salicyalic acid (5\% Salicylic acid in $\mathrm{H}_{2} \mathrm{SO}_{4}$ ) and $40 \mu \mathrm{L}$ of cell free supernatant was added and vortexed. The tubes were incubated in dark for $10 \mathrm{~min}$. The reaction was stopped by addition of $2 \mathrm{~mL}$ of $4 \mathrm{~N} \mathrm{NaOH}$. Optical density of this solution was measured after $20 \mathrm{~min}$ at 420 nm. The O.D was then compared to the standard curve prepared with known concentrations of $\mathrm{NaNO}_{3}$ (100$1000 \mathrm{ppm}$ ) to determine the concentration of Nitrate remaining in the medium (Cataldo et al., 1975).

Phosphate removal: Phosphate can be detected by spectrophotometric method by conversion of the phosphates to Molybdophosphoric acid Complex (MOP) by Ammonium molybdate, followed by reduction of the MOP Complex by $\mathrm{Sn}^{2+}$ of $\mathrm{SnCl}_{2}$ to give a blue coloured complex. For our study, at first a standard curve of phosphate was prepared by using standard solutions of phosphate from 0.05-0.5 ppm. $10 \mathrm{~mL}$ of cell free supernatant of bacterial sample ( $2 \%$ inoculum grown in Nitrate broth for $16 \mathrm{hrs}$ at $37^{\circ} \mathrm{C}$ ) was diluted in $60 \mathrm{~mL}$ of distilled water in a $250 \mathrm{~mL}$ conical flask. $2 \mathrm{~mL}$ of Ammonium molybdate reagent was added followed by 4 drops of Stannous chloride. The solution was shaken well and volume was made upto $100 \mathrm{~mL}$. The blue colour which developed indicated presence of phosphate which could be measured spectrophotometrically at $660 \mathrm{~nm}$. The unknown concentration of phosphate was determined by comparing with the standard curve (Krishnaswamy et al., 2009).

Statistical analysis: The objective of the study being isolation of efficient nitrate and phosphate removers, the ideal situation would be a single isolate performing both the functions. The relation between nitrate and phosphate removal was investigated by using the Correlation Co-efficient as a measure of the association between the two variables. The correlation co-efficient measures the strength of the linear relationship between the variables.

\section{RESULTS}

Environmental parameters: The physical parameters assessed were $\mathrm{pH}$, Suspended solids, Turbidity level of the water body, Temperature, Odour and Colour (Table 2).

The Chemical parameters include Alkalinity, Hardness, Calcium, Magnesium, Chlorine, Flouride, Nitrate, Ammonia, Phosphate, Total Iron, Residual Chlorine, Biological Oxygen Demand (Table 3).

The biological parameters were assessed by the growth of microbes in four different kinds of medium HiChrome E.coli coliform Selective agar base (HiMedia M-1294 for Escherichia coli, Enterobacter aerogenes, Shigella flexneri, Klebsiella pneumonia); HiChrome UTI agar base (HiMedia M1353 for Pseudomonas aeruginosa, Proteus mirabilis, Enterococcus faecalis); Salmonella differential agar base (HiMedia M-1078 for Salmonella $s p$ ); and HiChrome Aureus agar base (HiMedia M-1468 for Staphylococcus epidermidis and Listeria monocytogenes) (Table 4).

Cultivation medium and growth conditions: The serial dilution of water samples from the above mentioned 30 sites on medium with 2000 ppm of nitrate gave 130 different colonies. Further selection was made on the basis of morphology since most of the nitrate reducers according to literature are bacilli therefore bacilli in long or short chain or isolated bacilli were selected. 
OnLine J. Biol. Sci., 12 (2): 62-71, 2012

Table 2: Water samples were collected from 28 different environmental sites which were then tested for different physical parameters like pH, suspended solids, turbidity level, Temperature, Odour and Colour

\begin{tabular}{|c|c|c|c|c|c|c|}
\hline SITES & $\mathrm{pH}$ & $\begin{array}{l}\text { Susp.solid } \\
(\mathrm{gm})\end{array}$ & $\begin{array}{l}\text { Turbidity } \\
\text { level }(\mathrm{cm})\end{array}$ & $\begin{array}{l}\text { Temp } \\
\left({ }^{\circ} \mathrm{C}\right)\end{array}$ & Odour & Colour \\
\hline Udaypur Beach (West Bengal) & 6.30 & 0.008 & Not applicable & 15.0 & Fishy & Normal \\
\hline Digha Beach (West Bengal) & 6.10 & 0.063 & Not applicable & 15.0 & Normal & Normal \\
\hline Mandarmani (West Bengal) & 5.70 & 0.056 & Not Applicable & 15.0 & Normal & Normal \\
\hline Haldiram fountain & 7.02 & 0.015 & Not applicable & 24.0 & Fishy & Normal \\
\hline Baguiati Fountain & 7.25 & 0.030 & Not applicable & 24.0 & Fishy & Normal \\
\hline Ganga (Naihati) & 7.42 & 0.010 & 5.1 & 21.0 & Normal & Off white \\
\hline Ichamati (Taki) & 6.50 & 0.005 & 18.1 & 21.0 & Unpleasant & dirty light brown \\
\hline Churni (Ranaghat) & 6.50 & 0.009 & 55.1 & 21.0 & Normal & normal \\
\hline Damodar (Bardhaman) & 7.10 & 0.068 & 17.75 & 22.5 & Normal & Normal \\
\hline Mayurakshi (Tilpara) & 7.50 & 0.013 & Not applicable & 20.0 & Normal & Normal \\
\hline Agnikundu, Bakreswar & 8.84 & 0.002 & 100 & 72.0 & Normal & Normal \\
\hline Dudhkundu, Bakreswar & 7.40 & 0.001 & 100 & 31.5 & Fishy & Normal \\
\hline Jibatsakundu, Bakreswar & 7.20 & 0.003 & 100 & 22.0 & Normal & Normal \\
\hline Swet ganga (Birbhum) & 7.90 & 0.017 & 28.3 & 34.0 & Normal & Normal \\
\hline Taptapani (Orissa) & 7.30 & 0.016 & 100 & 40.0 & Normal & Normal \\
\hline West Bengal state university (Barasat) & 6.40 & 0.018 & Not applicable & 22.0 & Normal & Light brown \\
\hline Rhizosphere of aquatic plants & 7.10 & 0.003 & Not applicable & 22.0 & Normal & Normal \\
\hline Under Ultadanga over bridge & 7.20 & 0.054 & 20.5 & 21.0 & Fishy & Green \\
\hline Byepass Kalyani Highway(Fordillapur) & 7.30 & 0.014 & 18.1 & 22.0 & Normal & Green \\
\hline Site reserved for Himangini & 7.60 & 0.028 & 49.5 & 22.0 & Fishy & Very light green \\
\hline Jute Retting Site Thankurbari & 6.50 & 0.066 & 5.2 & 22.0 & Unpleasant & Black \\
\hline Kaikhali pond & 7.80 & 0.006 & 49.0 & 23.0 & Normal & Normal \\
\hline Rajarhat pond & 7.30 & 0.014 & 5.1 & 20.0 & Normal & Green \\
\hline Captain Bheri & 7.20 & 0.028 & 28.1 & 21.0 & Normal & Green \\
\hline Private Bheri & 7.30 & 0.036 & 20.5 & 24.0 & Normal & Light green \\
\hline Kestopur khal & 6.50 & 0.011 & 22.0 & 21.5 & Unpleasant & Black \\
\hline Bagjola khal & 6.80 & 0.043 & 18.1 & 21.0 & Unpleasant & Grey \\
\hline Khal Next to Captain Bheri & 6.50 & 0.059 & 20.0 & 23.0 & Unpleasant & Grey \\
\hline
\end{tabular}

Table 3: Water samples were collected from 28 different environmental sites which were then tested for different chemical parameters of the sampling sites like Alkalinity, Hardness, Calcium, Magnesium, Chlorine, Fluorine, Nitrate, Ammonia, phosphate, total Iron, Residual Chlorine and Biological Oxygen Demand in parts per million (ppm)

\begin{tabular}{|c|c|c|c|c|c|c|c|c|c|c|c|c|}
\hline SITES & $\begin{array}{l}\text { Alk } \\
\text { (ppm) }\end{array}$ & $\begin{array}{l}\text { Hard } \\
(\mathrm{ppm})\end{array}$ & $\begin{array}{l}\mathrm{Ca} \\
(\mathrm{ppm})\end{array}$ & $\begin{array}{l}\mathrm{Mg} \\
(\mathrm{ppm})\end{array}$ & $\begin{array}{l}\mathrm{Cl} \\
(\mathrm{ppm})\end{array}$ & $\begin{array}{l}\text { F } \\
(\mathrm{ppm})\end{array}$ & $\begin{array}{l}\text { NO3 } \\
\text { (ppm) }\end{array}$ & $\begin{array}{l}\text { NH3 } \\
\text { (Ppm) }\end{array}$ & $\begin{array}{l}\mathrm{PO} 4 \\
(\mathrm{Ppm})\end{array}$ & $\begin{array}{l}\text { Total Iron } \\
\text { (ppm) }\end{array}$ & $\begin{array}{l}\text { Res Cl } \\
\text { (ppm) }\end{array}$ & $\begin{array}{l}\text { BOD } \\
\text { (ppm) }\end{array}$ \\
\hline Udaypur Beach (West Bengal) & 88 & 7400 & 4070.4 & 809.090 & 15095.320 & 1.5 & 17.73 & 0.5 & 0.15 & 0.00 & 0.00 & 2.4 \\
\hline Digha Beach (West Bengal) & 96 & 7216 & 4032.0 & 773.710 & 15295.250 & 1.0 & 19.02 & 0.5 & 0.16 & 0.00 & 0.00 & 2.0 \\
\hline Mandarmani (West Bengal) & 88 & 1248 & 793.0 & 110.565 & 8678.000 & 1.5 & 19.30 & 0.5 & 0.04 & 0.00 & 0.00 & 2.8 \\
\hline Haldiram fountain & 340 & 360 & 112.0 & 60.260 & 207.080 & 1.0 & 0.00 & 0.0 & 0.20 & 0.00 & 0.10 & 2.0 \\
\hline Baguiati Fountain & 180 & 240 & 92.8 & 35.760 & 262.410 & 1.5 & 8.28 & 0.0 & 0.00 & 0.00 & 0.10 & 6.6 \\
\hline Naihati (Ganga) & 164 & 184 & 54.4 & 31.490 & 33.980 & 3.0 & 20.89 & 0.0 & 1.57 & 0.20 & 0.00 & 0.0 \\
\hline Taki (Ichamati) & 180 & 600 & 115.2 & 117.800 & 1099.659 & 1.5 & 22.73 & 0.0 & 0.00 & 0.00 & 0.00 & 4.4 \\
\hline Ranaghat(Churni) & 348 & 360 & 112.0 & 60.260 & 19.990 & 2.0 & 16.71 & 0.0 & 0.00 & 0.00 & 0.00 & 1.2 \\
\hline Bakreswar (Damodar) & 108 & 120 & 48.0 & 17.490 & 45.980 & 1.5 & 29.70 & 0.0 & 0.609 & 0.00 & 0.00 & 1.2 \\
\hline Tilpara(Mayurakshi) & 72 & 200 & 44.8 & 37.710 & 13.990 & 1.5 & 25.60 & 0.0 & 0.00 & 0.00 & 0.00 & 2.4 \\
\hline Agnikundu, & 124 & 8 & 0.0 & 1.940 & 52.868 & 1.0 & 32.90 & 0.0 & 0.00 & 0.00 & 0.00 & 0.8 \\
\hline Dudhkundu, & 140 & 80 & 16.0 & 15.550 & 129.950 & 1.0 & 29.76 & 0.0 & 1.54 & 0.20 & 0.00 & 1.6 \\
\hline Jibatsakundu, & 180 & 104 & 48.0 & 13.60 & 129.950 & 0.0 & 33.93 & 0.0 & 0.00 & 0.20 & 0.00 & 2.0 \\
\hline Swet ganga (Birbhum) & 140 & 40 & 9.6 & 7.38 & 121.960 & 1.0 & 17.73 & 0.0 & 0.14 & 0.20 & 0.00 & 2.8 \\
\hline Taptapani (Orissa) & 140 & 480 & 41.6 & 106.53 & 23.990 & 0.5 & 16.15 & 0.0 & 0.02 & 0.00 & 0.00 & 1.6 \\
\hline West Bengal state university (Barasat) & 228 & 176 & 64.0 & 27.21 & 154.950 & 0.5 & 19.95 & 0.5 & 0.00 & 0.00 & 0.00 & 1.2 \\
\hline Rhizosphere of aquatic plants & 172 & 200 & 51.2 & 36.15 & 119.960 & 1.5 & 20.23 & 0.5 & 0.45 & 0.20 & 0.00 & 2.8 \\
\hline Ultadanga over bridge & 404 & 560 & 176.0 & 93.31 & 624.800 & 2.0 & 19.40 & 0.5 & 0.00 & 0.20 & 0.00 & 0.4 \\
\hline Byepass Kalyani Highway (Fordillapur) & 172 & 216 & 76.8 & 33.82 & 74.940 & 1.5 & 10.78 & 0.0 & 0.00 & 0.00 & 0.00 & 2.8 \\
\hline Site reserved for Himangini & 340 & 360 & 112.0 & 60.26 & 187.440 & 1.5 & 18.00 & 0.5 & 0.00 & 0.00 & 0.00 & 4.4 \\
\hline Jute Retting Site Thankurbari & 320 & 240 & 96.0 & 34.99 & 49.980 & 2.0 & 22.00 & 0.5 & 2.22 & 0.20 & 0.10 & 2.8 \\
\hline Kaikhali pond & 280 & 224 & 51.2 & 41.99 & 54.980 & 1.5 & 18.00 & 0 & 0.00 & 0.20 & 0.00 & 7.2 \\
\hline Rajarhat pond & 356 & 360 & 89.6 & 65.70 & 149.950 & 2.0 & 27.45 & 0.5 & 1.04 & 0.40 & 0.10 & 2.4 \\
\hline Captain Bheri & 80 & 120 & 96.0 & 5.83 & 249.920 & 1.5 & 6.80 & 0.5 & 0.20 & 0.40 & 0.10 & 2.8 \\
\hline Private Bheri & 85 & 600 & 96.0 & 122.47 & 189.940 & 0.5 & 3.10 & 1.0 & 0.00 & 0.40 & 0.15 & 3.2 \\
\hline Kestopur khal & 168 & 192 & 64.0 & 31.10 & 87.470 & 1.5 & 15.00 & 0.0 & 0.33 & 0.20 & 0.00 & 0.4 \\
\hline Bagjola khal & 280 & 280 & 89.6 & 46.26 & 149.950 & 2.0 & 26.25 & 0.0 & 0.62 & 0.60 & 0.00 & 2.4 \\
\hline Khal Next to Captain Bheri & 200 & 384 & 131.2 & 61.43 & 252.40 & 1.0 & 10.00 & 0.5 & 1.50 & 0.80 & 0.00 & 2.0 \\
\hline
\end{tabular}

Alk-Alkalinity; Hard-Hardness; Ca-Calcium; Mg-Magnesium; $\mathrm{NO}_{3}$ - Nitrate; $\mathrm{PO}_{4^{-}}$Phosphate; Res Cl-Residual Chlorine; BOD- Biological Oxygen demand 


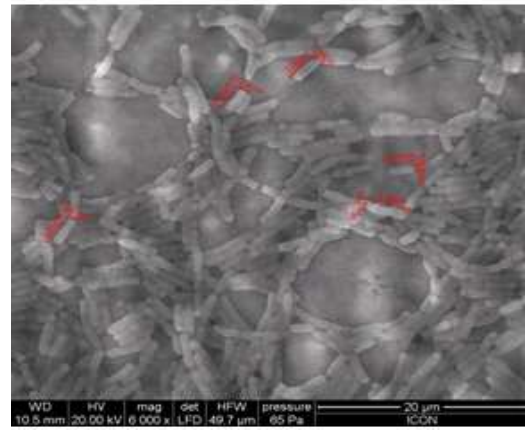

(a)

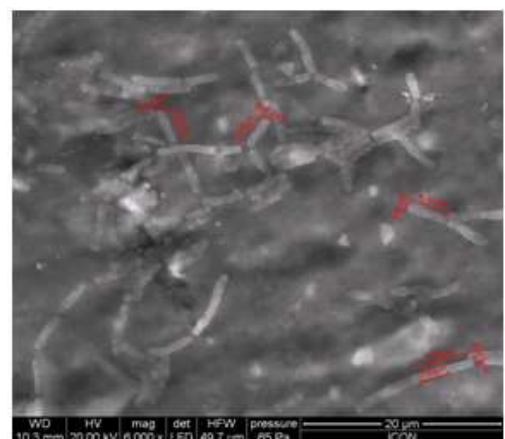

(d)

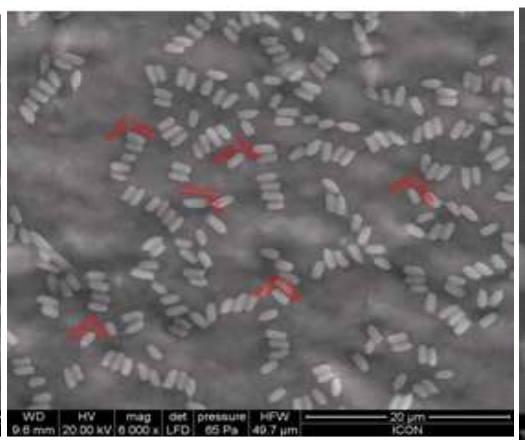

(b)

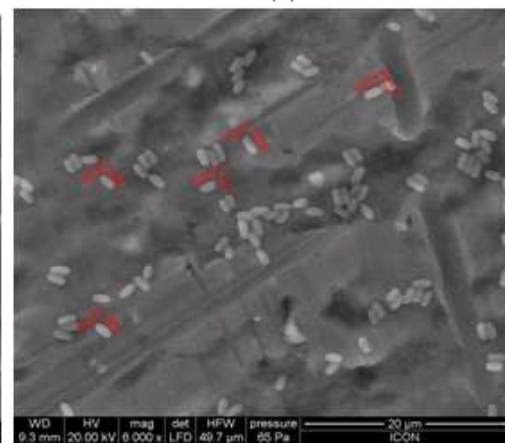

(e)

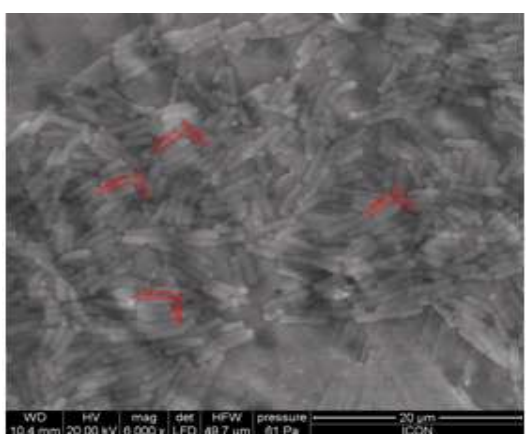

(g)

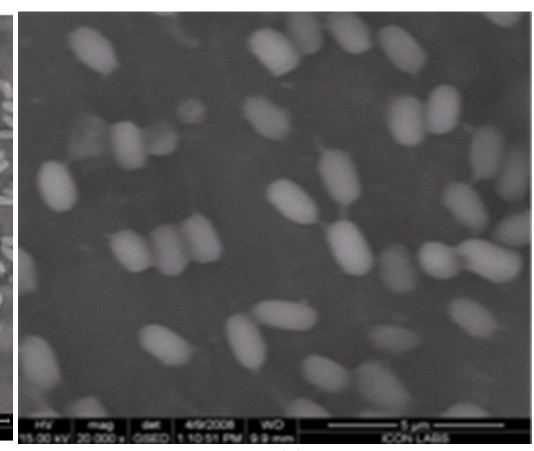

(c)

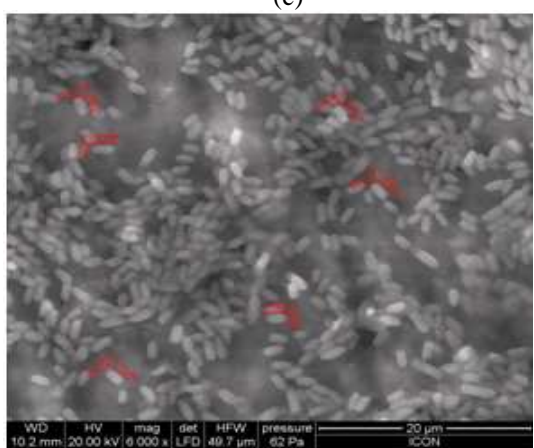

(f)

Fig. 1:(a) (WBUNB005) (b) WBUNB008 (c) SM2 (d) WBUNB006 (e) WBUNB009 (f) WBUNB004 (g) WBUNB007 The figures represent the Scanning electron micrographs of the isolates at different

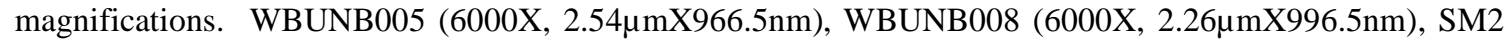

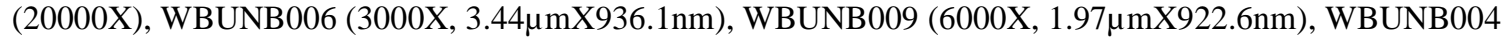
$(6000 \mathrm{X}, 1.95 \mu \mathrm{mX} 923.3 \mathrm{~nm})$, and WBUNB007 $(6000 \mathrm{X}, 3.18 \mu \mathrm{mX} 791.6 \mathrm{~nm})$ These were visualized by Scanning electron microscope (FEI QUANTA 200 MARK 2)

19 such strains were then checked for their efficiency of nitrate removal from liquid culture. 7 bacilli were selected on the basis of nitrate removing efficiency for further characterization.

Morphological characterization: Simple staining of the microbes showed different morphology of microbes. The Environmental Scanning Electron Micrographs of the isolates (Fig. 1) show that WBUNB004, WBUNB005 and WBUNB006 (from rhizosphere of water lily) are bacilli in chain whereas WBUNB008 (from paddy field), SM2 (from marine beach) and WBUNB009 (from raw sewage canal) are short isolated bacilli and WBUNB007 (from marine beach) is long bacilli.Gram staining showed that all the isolates were gram positive in nature.

Biochemical characterization: The biochemical characterization of the strains is represented in Table 5. All isolates except WBUNB007 produced oxidase and protease. 
OnLine J. Biol. Sci., 12 (2): 62-71, 2012

Table 4:Water samples were collected from 28 different environmental sites which were then tested for various microbial populations by incubating diluted water samples on medium specific for the growth of Escherichia coli, Enterobacter aerogenes, Shigella flexneri, Klebsiella pneumonia, Pseudomonas aeruginosa, Proteus mirabilis, Enterococcus faecalis, Salmonella sp. and Staphylococcus aureus

\begin{tabular}{|c|c|c|c|c|c|c|c|c|c|c|}
\hline SITES & $\begin{array}{l}\text { E.coli } \\
\text { It }\end{array}$ & $\begin{array}{l}\text { E. } \\
\text { Aerogel }\end{array}$ & $\begin{array}{ll} & S . \\
\text { enes } & \text { Flexner }\end{array}$ & $\begin{array}{ll} & \text { K. } \\
\text { ri } & \text { Pneumonic }\end{array}$ & $\begin{array}{cl} & \text { P.aer } \\
\text { ia } & \text { uginosa }\end{array}$ & $\begin{array}{l}\text { P.mir } \\
\text { abilis }\end{array}$ & $\begin{array}{l}\text { E.fae } \\
\text { calis }\end{array}$ & Salmonella sp & $p$ S. epide & $\begin{array}{l}\text { L.monocy } \\
\text { togenes }\end{array}$ \\
\hline Udaypur Beach (West Bengal) & 0 & 20 & 0 & 0 & 0 & 0 & 0 & 0 & 0 & 0 \\
\hline Digha Beach (West Bengal) & 80 & 160 & 740 & 60 & 320 & 0 & 100 & 20 & 20 & 40 \\
\hline Mandarmani (West Bengal) & 0 & 0 & 13 & 40 & 27 & 0 & 40 & 0 & 0 & 13 \\
\hline Haldiram fountain & 4000 & 2480 & 3120 & 6000 & 114000 & 0 & 0 & 0 & 40 & 160 \\
\hline Baguiati fountain & 2000 & 200 & 3600 & 100 & 160 & 0 & 160 & 200 & 0 & 0 \\
\hline Naihati (Ganga) & 300 & 420 & 2400 & 220 & 960 & 0 & 720 & 80 & 400 & 0 \\
\hline Taki (Ichamati) & 120 & 280 & 800 & 320 & 2400 & 0 & 160 & 1040 & 2480 & 0 \\
\hline Ranaghat(Churni) & 0 & 0 & 0 & 160 & 60 & 0 & 20 & 80 & 0 & 0 \\
\hline Bakreswar (Damodar) & 1020 & 220 & 0 & 480 & 0 & 0 & 280 & 320 & 1000 & 0 \\
\hline Tilpara(Mayurakshi) & 160 & 940 & 120 & 200 & 420 & 0 & 720 & 40 & 0 & 0 \\
\hline Agnikundu, Bakreswar & 0 & 0 & 0 & 0 & 0 & 0 & 0 & 0 & 20 & 0 \\
\hline Dudhkundu, Bakreswar & 1200 & 3680 & 240 & 80 & 800 & 0 & 2480 & 3300 & 0 & 0 \\
\hline Jibatsakundu, Bakreswar & 2400 & 4800 & 800 & 800 & 160 & 400 & 3280 & 160 & 280 & 140 \\
\hline Swet ganga (Birbhum) & 240 & 0 & 40 & 80 & 420 & 0 & 260 & 60 & 0 & 40 \\
\hline Taptapani (Orissa) & 0 & 0 & 17760 & 0 & 20560 & 0 & 0 & 0 & 0 & 0 \\
\hline West Bengal state university (Barasat) & 0 & 200 & 1300 & 140 & 900 & 0 & 240 & 2740 & 0 & 60 \\
\hline Rhizosphere of aquatic plants & 1600 & 0 & 0 & 540 & 1040 & 0 & 160 & 800 & 0 & 20 \\
\hline Under Ultadanga over bridge & 150000 & 1600 & 0 & 10000 & 66000 & 0 & 42000 & 50000 & 0 & 1920 \\
\hline Byepass Kalyani Highway (Fordillapur) & 440 & 300 & 0 & 140 & 0 & 0 & 0 & 0 & 160 & 0 \\
\hline Site reserved for Himangini & 40000 & 1120 & 0 & 800 & 320 & 0 & 0 & 0 & 0 & 1560 \\
\hline Jute Retting Site Thankurbari & 312000 & 40000 & 112000 & 48000 & 56000 & 0 & 24000 & 40000 & 100 & 220 \\
\hline Kaikhali pond & 80 & 120 & 220 & 100 & 0 & 0 & 380 & 20 & 0 & 120 \\
\hline Rajarhat pond & 4000 & 1740 & 0 & 8000 & 0 & 0 & 0 & 4000 & 0 & 0 \\
\hline Captain Bheri & 1800 & 520 & 8800 & 160 & 300 & 0 & 2120 & 0 & 0 & 0 \\
\hline Private Bheri & 800 & 1400 & 500 & 1200 & 1200 & 0 & 1120 & 0 & 0 & 1440 \\
\hline Kestopur khal & 72000 & 32000 & 64000 & 72000 & 90000 & 0 & 192000 & 48000 & 140 & 420 \\
\hline Bagjola khal & 240000 & 464000 & 88000 & 16000 & 208000 & 0 & 192000 & 0 & 2600 & 1340 \\
\hline Khal Next to Captain Bheri & 328000 & 0 & 56000 & 64000 & 272000 & 0 & 320000 & 0 & 2560 & 5760 \\
\hline
\end{tabular}

Table 5:Morphological and biochemical characterization of 7 isolates. The morphological characterization includes the Gram nature whereas the Biochemical Characterization includes the capability of the isolates to produce enzymes such as Catalase, Oxidase, Protease, Amylase, lipase and DNase

\begin{tabular}{|c|c|c|c|c|c|c|c|}
\hline Strain & Gram nature & Catalase & Oxidase & Protease & Amylase & Lipase & Dnase \\
\hline WBUNB005 & Gram positive bacilli & + & + & + & + & - & - \\
\hline WBUNB008 & Gram positive bacilli & - & + & + & + & - & + \\
\hline SM2 & Gram positive bacilli & + & + & + & + & - & + \\
\hline WBUNB006 & Gram positive bacilli & + & + & + & + & + & - \\
\hline WBUNB009 & Gram positive bacilli & + & + & + & + & + & + \\
\hline WBUNB004 & Gram positive bacilli & + & + & + & + & + & + \\
\hline WBUNB007 & Gram positive bacilli & - & - & - & + & - & + \\
\hline
\end{tabular}

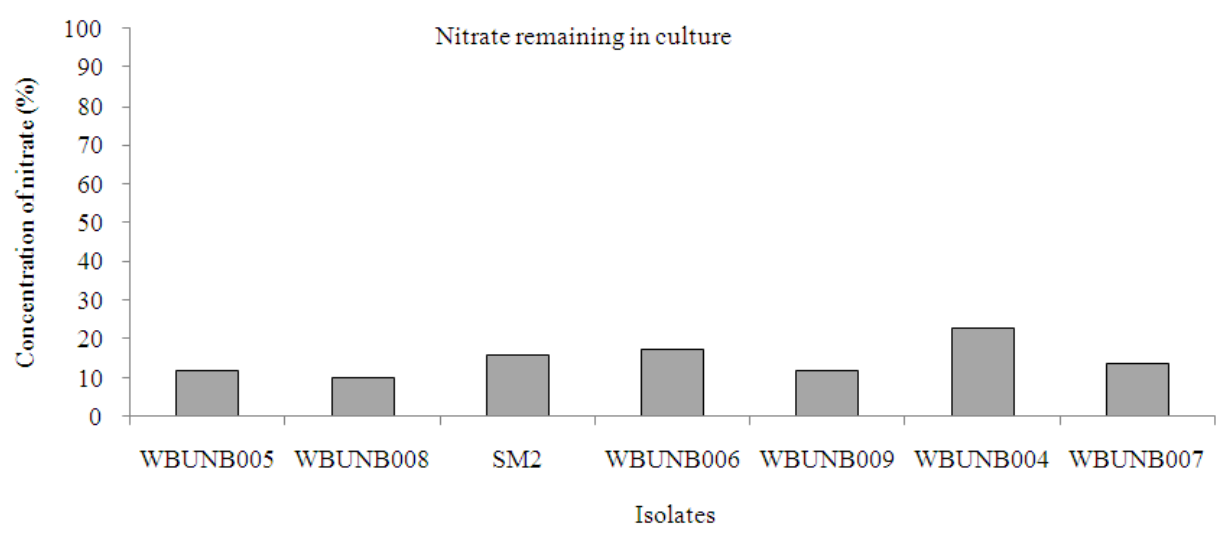

Fig. 2: Graph representing the percentage of nitrate remaining in the medium after incubation with the isolate for $16 \mathrm{~h}$ 
OnLine J. Biol. Sci., 12 (2): 62-71, 2012

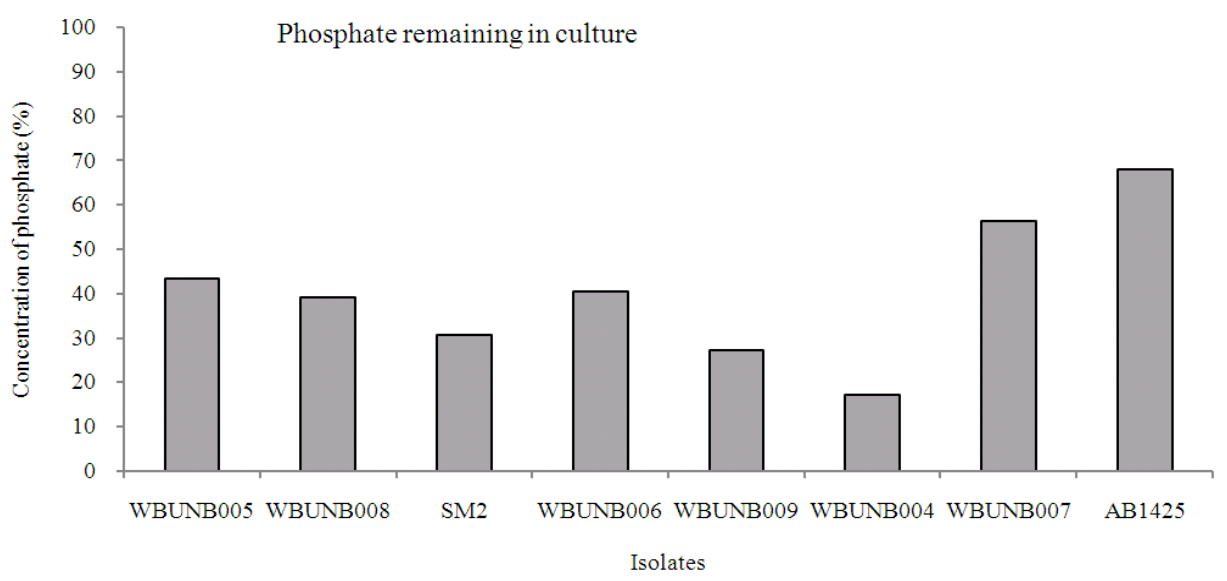

Fig. 3: Graph representing the percentage of phosphate remaining in the medium after incubation with the isolate for $16 \mathrm{~h}$

Table 6: 7 isolates were tested for the preference of the substrate they required for growth by using Hi-Media Carbohydrate kit (KB 009). The substrate preference of the isolates are given below

\begin{tabular}{|c|c|}
\hline STRAIN & SUBSTRATE UTILISED \\
\hline WBUNB005 & $\begin{array}{l}\text { Maltose, Fructose, Dextrose, Trehalose, Sucrose, LArabinose, Mannose, Glycerol, Rhamnose, Cellobiose, Melezitose, } \alpha \\
\text { Methylmannoside, Xylitol, Esculin, D-Arabinose, Citrate, Malonate, Sorbose }\end{array}$ \\
\hline WBUNB008 & $\begin{array}{l}\text { Maltose, Fructose, Dextrose, Galactose, Raffinose, Trehalose, Melibiose, Sucrose, Mannose, L-arabinose, Glycerol, Ribose } \\
\text { Rhamnose, Cellobiose, Melezitose, } \alpha \text { Methyl mannoside, Xylitol, Esculin, D-Arabinose, Citrate, Malonate, Sorbose }\end{array}$ \\
\hline SM2 & Maltose, Fructose, Dextrose, Trehalose, $\alpha$ Methyl-D-glucoside, Esculin \\
\hline WBUNB006 & $\begin{array}{l}\text { Maltose, Dextrose, Galactose, Raffinose, Trehalose, Melibiose, Sucrose, LArabinose, Mannose, Glycerol, Ribose Rhamnose, } \\
\text { Cellobiose, Melezitose, } \alpha \text { Methyl mannoside, Xylitol, Esculin, D-Arabinose, Citrate, Malonate, Sorbose }\end{array}$ \\
\hline WBUNB009 & $\begin{array}{l}\text { Maltose, Fructose, Dextrose, galactose, Raffinose, trehalose, Melibiose, Sucrose, Glycerol, Ribose Rhamnose, Cellobiose, } \\
\text { Melezitose, } \alpha \text { Methyl mannoside, Xylitol, Esculin, D-Arabinose, Citrate, Malonate, Sorbose }\end{array}$ \\
\hline WBUNB004 & $\begin{array}{l}\text { Maltose, Raffinose, Melibiose, L-Arabinose, Mannose, Ribose Rhamnose, Cellobiose, Melezitose, } \alpha \text { Methyl mannoside, } \\
\text { Xylitol, ONPG, Esculin, D-Arabinose, Citrate, Malonate, Sorbose }\end{array}$ \\
\hline WBUNB007 & $\begin{array}{l}\text { Dextrose, Trehalose, Glycerol, Ribose Rhamnose, Cellobiose, Melezitose, } \alpha \text { Methyl mannoside, Esculin, D } \\
\text { Citrate, Malonate, Sorbose }\end{array}$ \\
\hline
\end{tabular}

Table 7: The 7 isolates were tested for their resistance and susceptibility to a common range of antibiotics. The antibiotic susceptibility profile of the isolates is given below

\begin{tabular}{|c|c|c|c|c|c|c|c|c|}
\hline Antibiotics & Symbol & $\begin{array}{l}\text { Wbunb } \\
005\end{array}$ & $\begin{array}{l}\text { Wbunb } \\
008\end{array}$ & $\begin{array}{l}\text { SM } \\
2 \\
\end{array}$ & $\begin{array}{l}\text { Wbunb } \\
006\end{array}$ & $\begin{array}{l}\text { Wbunb } \\
009\end{array}$ & $\begin{array}{l}\text { Wbunb } \\
004\end{array}$ & $\begin{array}{l}\text { Wbunb } \\
007\end{array}$ \\
\hline Chloramphenicol (30 mcg) & C 30 & $\mathrm{~S}$ & $\mathrm{~S}$ & $\mathrm{I}$ & $\mathrm{S}$ & $\mathrm{S}$ & $\mathrm{S}$ & $\mathrm{S}$ \\
\hline Ceftazidime (30 mcg) & CA 30 & $\mathrm{R}$ & $\mathrm{R}$ & $\mathrm{R}$ & $\mathrm{R}$ & $\mathrm{R}$ & $\mathrm{R}$ & $\mathrm{R}$ \\
\hline Ampicillin (10 mcg) & A 10 & $\mathrm{R}$ & $\mathrm{R}$ & $\mathrm{R}$ & $\mathrm{R}$ & $\mathrm{R}$ & $\mathrm{R}$ & $\mathrm{R}$ \\
\hline Methicillin (4mcg) & MT 4 & $\mathrm{R}$ & $\mathrm{R}$ & $\mathrm{R}$ & $\mathrm{R}$ & $\mathrm{R}$ & $\mathrm{R}$ & $\mathrm{R}$ \\
\hline Rifampicin (15 mcg) & R 15 & $\mathrm{R}$ & $\mathrm{R}$ & $\mathrm{R}$ & $\mathrm{R}$ & $\mathrm{I}$ & $\mathrm{R}$ & $\mathrm{R}$ \\
\hline Norfloxacin (10 mcg) & N 10 & $\mathrm{~S}$ & $\mathrm{~S}$ & $\mathrm{~S}$ & $\mathrm{~S}$ & $\mathrm{~S}$ & $\mathrm{~S}$ & $\mathrm{~S}$ \\
\hline Roxithromycin & Ro & $\mathrm{S}$ & $\mathrm{S}$ & I & $\mathrm{S}$ & $\mathrm{S}$ & $\mathrm{S}$ & $\mathrm{S}$ \\
\hline Trimethoprim (5 mcg) & $\operatorname{Tr}$ & $\mathrm{R}$ & $\mathrm{R}$ & $\mathrm{R}$ & $\mathrm{R}$ & $\mathrm{R}$ & $\mathrm{R}$ & $\mathrm{R}$ \\
\hline Doxycycline Hydrochloride (30mcg) & DO 30 & $\mathrm{~S}$ & $\mathrm{~S}$ & $\mathrm{~S}$ & $\mathrm{~S}$ & $\mathrm{~S}$ & $\mathrm{~S}$ & $\mathrm{~S}$ \\
\hline Vancomycin (30 mcg) & VA 30 & I & I & I & I & I & I & $\mathrm{R}$ \\
\hline Cloxacillin (10 mcg) & CX 10 & $\mathrm{R}$ & $\mathrm{R}$ & $\mathrm{R}$ & $\mathrm{R}$ & $\mathrm{R}$ & $\mathrm{R}$ & $\mathrm{R}$ \\
\hline Polymyxin-B (300 units) & PB 300 & $\mathrm{R}$ & $\mathrm{R}$ & $\mathrm{R}$ & $\mathrm{R}$ & $\mathrm{R}$ & $\mathrm{R}$ & $\mathrm{R}$ \\
\hline Gentamycin (10 mcg) & G 10 & $\mathrm{~S}$ & $\mathrm{~S}$ & $\mathrm{~S}$ & $\mathrm{~S}$ & $\mathrm{~S}$ & $\mathrm{~S}$ & $\mathrm{~S}$ \\
\hline Ciprofloxacin (5 mcg) & CF 5 & $\mathrm{~S}$ & $\mathrm{~S}$ & $\mathrm{~S}$ & $\mathrm{~S}$ & $\mathrm{~S}$ & $\mathrm{~S}$ & $\mathrm{~S}$ \\
\hline Cefotaxime $(30 \mathrm{mcg})$ & CTX 30 & $\mathrm{R}$ & I & I & $\mathrm{R}$ & $\mathrm{R}$ & $\mathrm{R}$ & $\mathrm{R}$ \\
\hline Cephadroxil (30 mcg) & CQ 30 & $\mathrm{~S}$ & $\mathrm{~S}$ & I & $\mathrm{S}$ & $\mathrm{S}$ & $\mathrm{S}$ & $\mathrm{S}$ \\
\hline Teicoplanin (30 mcg) & TE 30 & $\mathrm{~S}$ & $\mathrm{~S}$ & $\mathrm{~S}$ & $\mathrm{~S}$ & $\mathrm{~S}$ & $\mathrm{~S}$ & $\mathrm{~S}$ \\
\hline Neomycin $(30 \mathrm{mcg})$ & N 30 & $\mathrm{~S}$ & $\mathrm{~S}$ & I & I & $\mathrm{I}$ & $\mathrm{R}$ & $\mathrm{R}$ \\
\hline
\end{tabular}


All except WBUNB007 and WBUNB008 produced catalase. Amylase is produced by all the isolates, whereas lipase is produced by WBUNB006, WBUNB009 and WBUNB004 and DNase by WBUNB008, SM2, WBUNB009 and WBUNB004. The substrate utilization profile of the isolates was performed by using different carbohydrate sources (Himedia KB009). This study gives us an array of substrates used by an isolate. Most of them could grow in presence of Maltose, Dextrose, Trehalose, Xylitol, Cellobiose, $\alpha$-Methyl Mannoside, Melezitose, DArabinose, Citrate, Malonate and Sorbose (Table 6).

The antibiotic susceptibility profile of the isolates were performed by using different antibiotic discs (Himedia). This study shows that the strains were resistant to antibiotics like Ceftazidime, Ampicillin, Methicillin, Rifampicin, Trimethoprim, Polymixin-B and Cefotaxime and sensitive to antibiotics like Chloramphenicol, Norfloxacin, Roxithromycin, Doxycycline hydrochloride, Gentamycin, Ciprofloxacin, Cefadroxil and Teicoplanin (Table 7).

Nitrate removal: The nitrate removal from the medium is the primary step for the reduction of nitrate though after removal the bacteria may use the nitrate by assimilatory or dissimilatory pathway. The result is represented in the form of percentage of nitrate remaining in the medium after incubation with the isolate for $16 \mathrm{~h}$ at $37^{\circ} \mathrm{C}$ (Fig. 2).

Phosphate removal: The phosphate removal capacities of the isolates were checked in enriched medium in comparison with a type strain, Acinetobacter baumanii (MTCC 1425) known for phosphate removal obtained from MTCC. The result is represented in the form of percentage of phosphate remaining in the medium after incubation with the isolate for $16 \mathrm{hrs}$ in $37^{\circ} \mathrm{C}$. The result indicates that all the isolates show better phosphate removal than Acinetobacter baumanii under the given set of conditions (Fig. 3).

Statistical analysis: The nitrate removal by the isolates were found to be within $77-88 \%$, the average removal being $85.3 \%$. The phosphate removal by the isolates were found to be within $43.8-82.9 \%$, the average being $63.71 \%$ while that of the type strain under similar conditions showed $31.9 \%$ removal. The correlation study of nitrate and phosphate showed a negative moderate correlation of (-) 0.5584 which implies that an efficient nitrate remover is not necessarily an efficient phosphate remover.

\section{DISCUSSION}

In this study we report the isolation of 7 strains with potential for nitrate removal. They could be used for bioremediation of nitrate contaminated sites leading to environmental protection. Phosphate removers isolated during the study were found to be more efficient than the type strain (Acinetobacter baumanii) under identical conditions. Here we report 7 gram positive bacterial isolates which are highly efficient in phosphate removal. Since the mechanism of phosphate removal in bacteria leads to the intracellular accumulation of polyphosphate granules, these could be used as potential candidates for sequestration of phosphate from environmental sites.

\section{CONCLUSION}

The study is a successful attempt to isolate efficient nitrate and phosphate removing bacteria from various environmental sites for remediation of waste water by reducing the nitrate and phosphate load. In addition optimization of the waste water treatment parameters by these isolates in future could not only lead to environmental protection but also sequestration of essential plant growth nutrients from the waste which in turn could be re used.

\section{ACKNOWLEDGEMENT}

The group wishes to acknowledge the financial assistance received from Indian Council for Agricultural Research (ICAR), Govt. of India vide Grant No. GB-2019 dated 24th May 2011 and infrastructural facilities of West Bengal University of Technology, India for carrying out the work. The group is grateful to Mr.Sudip Sen, Mr.Gutam Das and Mr.Sanjib Mondal for their kind support during sampling. The group wishes to thank and acknowledge Prof A.R.Thakur, Prof T.B.Samanta and Dr.K.Ray for their invaluable inputs and suggestions during the work. The authors express their sincere gratitude towards Dr. A Bandopadhyay, ICAR; Dr. A.K. Saxena, Indian Agricultural Research Institute (IARI) and Prof. A.K. Tripathy, Benaras Hindu University, India for their valuable suggestion regarding the selection of environmental sites and parameters of analysis.

\section{REFERENCES}

Agrawal, G.D., 1999. Diffuse agricultural water pollution in India. Water Sci. Technol., 39: 33-47. DOI: 10.1016/S0273-1223(99)00030-X

Cataldo, D.A., M. Maroon, L.E. Schrader and V.L. Youngs, 1975. Rapid colorimetric determination of nitrate in plant tissue by nitration of salicylic acid. Commun. Soil Sci. Plant Anal., 6: 71-80. DOI: 10.1080/00103627509366547 
Eckford, R.E. and P.M. Fedorak, 2002. Planktonic nitrate-reducing bacteria and sulfate-reducing bacteria in some western Canadian oil field waters. J. Ind. Microb. Biotechnol., 29: 8392. DOI: 10.1038/sj.jim.7000274

Filintas, A., P. Dioudis, G. Stamatis, J. Hatzopoulos and T. Karyotis, 2008. Environmental assesment of Groundwater nitrate pollution from agricultural wastes and fertilizers in central Greece watersheds using remote sensing and GIS. Proceedings of the 3rd International Conference AQUA 2008 on: Water Science and Technology with Emphasis on Water and Climate, Oct. 16-19, Athens, Greece, pp: $1-10$.

Giupponi, C., B. Eiselt and P.F. Ghetti, 1999. A multicriteria approach for mapping risks of agricultural pollution for water resources: The Venice Lagoon watershed case study. J. Environ. Manage., 56: 259-269.

DOI: 10.1006/jema.1999.0283

Jin, Z., Y. Chen, F. Wang and N. Ogura, 2004. Detection of nitrate sources in urban groundwater by isotopic and chemical indicators, Hangzhou City, China. Environ. Geol., 45: 1017-1024. DOI: 10.1007/s00254-004-0962-y

Jorgensen, L.A., 1999. The cycling of nitrogen in the Danish agricultural sector and the loss to the environment. Water Sci. Technol., 39: 15-23. DOI: 10.1016/S0273-1223(99)00028-1

Kinoshita, T., T. Katoh, T. Suji, M. Kanada and A. Inoue, 2003. Downward movement of inorganic nitrogen in tea [Camellia sinensis] fields of yellow soil affected by different $\mathrm{N}$-fertilizer application. Japanese J. Soil Sci. Plant Nut., 74: 637-643.

Kotoski, J.E., 1997. Phosphorus minifact analysis sheet. Spring Harbor Environmental Magnet Middle School.

Krishnaswamy, U., M. Muthusamy and L. Perumalsamy, 2009. Studies on the efficiency of the removal of phosphate using bacterial consortium for the biotreatment of phosphate wastewater. Eur. J. Applied Sci., 1: 6-15.
Nakasone, H. and T. Yamamoto, 2004. The impacts of the water quality of the inflow water from tea fields on irrigation reservoir ecosystems. Paddy Water Environ., 2: 45-50. DOI: 10.1007/s10333-004-0039-2

Nandy, P., A.R. Thakur and S.R. Chaudhuri, 2007. Characterization of bacterial strains isolated through microbial profiling of urine samples. Online J. Biol. Sci., 7: 44-51. DOI: 10.3844/ojbsci.2007.44.51

Pinar, G., E. Duque, A. Haidour, J. Oliva and L. Sanchez-Barbero et al., 1997. Removal of high concentrations of nitrate from industrial wastewaters by bacteria. Applied Environ. Microbiol., 63: 2071-2073.

Pradyot, P., 1997. Hand Book of Environmental Analysis, Chemical pollutants in Air, Water, Soil and Solid Wastes. 2nd Edn., CRC/Lewis Publishers, ISBN-10: 0873719891, pp: 584.

Singleton, M.J., K.N. Woods, M.E. Conrad, D.J. DePaolo and P.E. Dresel, 2005. Tracking sources of unsaturated zone and groundwater nitrate contamination using nitrogen and oxygen stable isotopes at the hanford site, washington. Washington. Environ Sci. Technol., 10: 35633570. DOI: $10.1021 / \mathrm{es} 0481070$

Wakida, F.T. and D.N. Lerner, 2005. Non-agricultural sources of groundwater nitrate: A review and case study. Water Res., 39: 3-16. DOI: 10.1016/j.watres.2004.07.026

Wakida, F.T. and D.N. Lerner, 2006. Potential nitrate leaching to groundwater from house building. Hydrol. $\quad$ Process., 20: 2077-2081. DOI: 10.1002/hyp.6143

Whitmore, A.P., N.J. Bradbury and P.A. Johnson, 1992. Potential contribution of ploughed grassland to nitrate leaching. Agric. Ecosyst. Environ., 39: 221233. DOI: 10.1016/0167-8809(92)90056-H 